\title{
Progression of myopia in a natural cohort of Chinese children during COVID-19 pandemic
}

\author{
Dandan $\mathrm{Ma}^{1}$ - Shifei Wei ${ }^{1}$. Shi-Ming $\mathrm{Li}^{1} \cdot$ Xiaohui Yang $^{1} \cdot \mathrm{Kai} \mathrm{Cao}^{1} \cdot$ Jianping Hu${ }^{1} \cdot$ Sujie Fan $^{2} \cdot$ Lihua Zhang $^{2}$. \\ Ningli Wang ${ }^{1}$ (i)
}

Received: 13 March 2021 / Revised: 16 June 2021 / Accepted: 29 June 2021 / Published online: 21 July 2021

(c) The Author(s) 2021

\begin{abstract}
Purpose To determine myopia progression in children during the COVID-19 and the related factors associated with myopia. Methods All subjects underwent three-timepoint ocular examinations that were measured in July 2019, January, and August 2020. We compared the changes in uncorrected visual acuity (UCVA), mydriatic spherical equivalent (SE), and axial length (AL) between two periods (before and during COVID-19). A questionnaire was performed to investigate risk factors for myopia. Results Compared with before the COVID-19, the mean (S.D.) myopia progression during the COVID-19 was significantly higher in right eyes $(-0.93(0.65)$ vs. $-0.33(0.47) \mathrm{D} ; p<0.001)$. However, the differences in UCVA changes and the axial elongation between two periods were clinically insignificant. Through logistic regressive analysis, we found the difference of the SE changes was associated with the baseline AL ( $P=0.028$; 95\% confidence interval $[\mathrm{CI}], 1.058,2.632)$, online education $(P=0.02 ; 95 \% \mathrm{CI}, 1.587,8.665)$, and time of digital screen $(p<0.005 ; 95 \% \mathrm{CI}, 1.587,4.450)$.

Conclusions Children were at higher risk of myopia progression during COVID-19, which was associated with the baseline AL, the longtime online learning, and digital screen reading.
\end{abstract}

Keywords Myopia progression · Mydriatic spherical equivalent · COVID-19

\section{Key messages}

- Previous studies have demonstrated that home confinement during the COVID-19 outbreak may worsen the global burden of myopia.

- This is asignificant report to determinate the impact of myopia in a nature cohort of children during studying at home by comparing the cycloplegic spherical equivalent, uncorrected visual acuity and axial length, providing a new insight of myopia development and progression.

- Finally, we found that myopia progression was related to initial AL, longtime of online learning and longtime of digital screen.

- Studying at home during COVID-19 may be posing a threat to the development and progression of myopia in children and adolescents, especially in East Asia, where the prevalence of myopia is high.

Ningli Wang

wningli@vip.163.com

1 Beijing Ophthalmology \& Visual Sciences Key Laboratory, Beijing Institute of Ophthalmology, Beijing Tongren

Eye Center, Beijing Tongren Hospital, Capital Medical University, NO.1 Dongjiaominxiang Street, Dongcheng

District, Beijing 100730, China

2 Handan City Eye Hospital, Handan 056000, China

\section{Introduction}

The novel coronavirus disease 2019 has had a global impact [1] and affected almost every aspect of people's lives. As of January 1, 2021, more than 83.77 million have been confirmed case and 1.82 million have died globally [2]. For the first time in human history, global children could not go to 
school, but study at home because of the various lockdown measures imposed on populations everywhere to contain to spread of the virus. During the COVID-19 pandemic, governments around the world have provided deliver distance education at scale in an attempt to ensure continuity of learning. According to United Nations Educational, Scientific and Cultural Organization (UNESCO), as of early-September, 820 million children and youth were affected by school closures, from pre-primary to higher education. Forty-six countries were in a state of nationwide school suspension [3].

It was speculated that the closure of schools and study at home may have negative effects on children's physical and mental health [4]. The Ministry of Education of the People's Republic of China estimated that more than 220 million children and adolescents were kept at home and had online education in China [5]. During the COVID-19 pandemic, school was closed, but student learning was continuing online. It is necessary to investigate whether their near work increased, while outdoor activities decreased during studying at home. Students completed their learning through online learning, and the usage time of digital screen was prolonged. These effects on children may lead to the development and progression of myopia.

Myopia has become a significant public health problem. There are about 2.5 billion myopic people in the world [6]. Myopia is the most common cause of vision impairment in children [7], especially in Singapore [8], Japan [9], and China [10-13]. The international and domestic researchers were concerned about whether home confinement and studying at home during the COVID-19 pandemic worsen the global burden of myopia [14]. However, data on myopia ocular biometric parameters in children during studying at home were lacking.

From January 2020 to August 2020, schools were closed, and students completed deliver distance education at home. Ministry of Education of the People's Republic of China said that the rate of myopia among Chinese primary and middle school students increased by $11.7 \%$ in the first 6 months of the year [15]. It is urgent to investigate the development and progression of myopia in children and its influencing factors during studying at home compared with pre-outbreak. As far as we know, this is the significant report to determinate the impact of myopia in a nature cohort of children during studying at home by comparing the mydriatic spherical equivalent, uncorrected visual acuity, and axial length, providing a new insight of myopia development and progression.

\section{Methods}

\section{Studying cohort}

Studied population included, children aged 8 to 10 years with the best-corrected visual acuity at least Log MAR
VA 0.0 in both eyes in Handan of Hebei Province, China. Exclusion criteria were those with systemic disease or former or present eye disease or injury. Children with atropine or orthokeratology or any conditions that might have influenced myopia were excluded. A total of 208 children were examined three times, including ocular examinations and a questionnaire. Baseline data were collected in July 2019. Two follow-up visits were conducted in January 2020 and August 2020. The questionnaires were performed in August 2020.

In order to determine the change value of children's eye parameters in the 6 months before the COVID-19 outbreak, we set a control group. We included 83 children with myopia who were admitted to Beijing Tongren Hospital, China, from April 2018 to July 2018 and followed up for 6 months. All children met the inclusion criteria.

The research was approved by the Ethics Committee of Beijing Tongren Hospital, Capital Medical University. All participating students gave written informed consent and adhered the principles of the Declaration of Helsinki.

\section{Procedures}

Children underwent a comprehensive and standardized ocular examination, including uncorrected visual acuity, cycloplegic refraction, and axial length at baseline and two follow-up visits. In addition, a detailed questionnaire was conducted at the last follow-up.

All children were conducted for distance visual acuity (VA) without spectacles, using logarithmic visual acuity chart (Precision Vision, La Salle, IL, USA) at a distance of $4 \mathrm{~m}$. The chart was retro-illuminated and has 70 tumbling "E" optotypes with five letters on each line. Children were examined monocularly (left eye followed by right eye); the detailed procedure has described elsewhere [16].

The autorefractor-keratometry (KR8800, Topcon, Tokyo, Japan) was used to measure after cycloplegic autorefraction. For each student, three drops of $1 \%$ cyclopentolate (Cyclogyl, Alcon-Convreur, Rijksweg, Belgium) were administered at interval of $5 \mathrm{~min}$ apart. If the pupil size was less than $6.0 \mathrm{~mm}$ after $30 \mathrm{~min}$, a fourth drop of $1 \%$ cyclopentolate was administered [17]. Three auto-refraction readings were taken, and the average was recorded.

The axial length was measured by the IOL master (Carl Zeiss Meditec AG, Jena, Germany). Five repeated measurements were detected and obtained the average AL value before cycloplegic.

The questionnaire used in our study was derived from Anyang Children Eye Study, and the detailed procedure has been described elsewhere [18]. To control interview bias, the questionnaire survey was performed a pilot study on the validity and reliability [12]. Generally, the questionnaire 
mainly focusses to collect subjects near work and outdoor activity-related parameters, including time (hours/day) and modes of near work and outdoor activities before and during studying at home. Near work included homework, reading books, painting, playing chess, using computer, and using mobile phone. Outdoor activities included bicycle riding, running, swimming, and playing football. The parents and children were asked the time (hours/day) and parameter of online education during study at home. All questionnaires were completed by children and parents at the same time.

\section{Definitions}

The spherical equivalent (SE) is calculated according to the standard formula of the algebraic sum of the dioptric powers of the sphere and half of the cylinder (sphere $+0.5 \times$ cylinder). Myopia was defined as SE $\leq-0.5 \mathrm{D}$ [19]. The emmetrope and hyperopia were defined as SE between $-0.5 \mathrm{D}$ and $+0.5 \mathrm{D}$, and great than $+0.5 \mathrm{D}$, respectively [20].

In period one (July 2019 to January 2020), the changes of subjects' refractive error were the changes within 7 months before outbreak, and we set them as the control period. In period two (January 2020 to August 2020), the changes of refractive error were the changes within 7 months during the COVID-19 pandemic study at home, and we set them as the experimental period. The difference of the SE's change between the control period and experimental period was defined as the change of SE in the period one minus the change of SE in the period two. The value of difference of the SE's change was classified as flat $(S E \geq 0 D)$ and decline $(\mathrm{SE}<0)$.

\section{Data management and statistical analysis}

The database used Epidata software 3.1 (The Epidata Association, Odense, Denmark), and statistical analysis was performed using SPSS 26.0 (IBM SPSS Statistics, Chicago, IL).

Baseline variable of ocular characteristics is described by maximum, minimum, mean, and standard deviation. Nonparametric tests were used to investigate statistical significance among control group, period one, and period two. Paired $t$ test that is used to conform to normal distribution and rank sum test that is used for non-normal distribution were used to determine statistical significance between the period one and period two. To detect potential risk factors of development of myopia, the binary logistic regression was used to analyze the influencing factors of eye parameters. Since large correlation coefficients for cycloplegic SE were observed between the two eyes $(r=0.73, P<0.001)$, only data from right eyes were included in the analyses. A twosided $P$-values less than 0.05 were considered statistically significant.

\section{Results}

A total 208 children completed examinations and were included for analysis. A total of 109 (52.4\%) subjects were male. The age range was $8-10$ years old with a mean age of $8.9 \pm 0.69$ years. Among the 208 children included in the study, 90 were myopia, 77 were emmetropia, and 41 were hyperopia. Ocular characteristics at baseline are presented in Table 1 . The mean uncorrected visual acuity of right eyes was $0.19 \pm 0.22$. The mean axial length and spherical equivalent of right eyes were $23.08 \pm 0.92 \mathrm{~mm}$ and $-0.50 \pm 1.25 \mathrm{D}$.

Among the 83 children in the control group, 38 children were myopia, 25 children were emmetropia, and 20 children were hyperopia. The mean axial length was $23.85 \pm 0.94 \mathrm{~mm}$ in the control group and $23.08 \pm 0.91 \mathrm{~mm}$ in the study group. There was no statistical difference in axial length between the two groups $(P>0.05)$. The mean SE of the control group and the study group were $-0.47 \pm 1.38 \mathrm{D}$ and $-0.50 \pm 1.25$ $\mathrm{D}$, respectively $(P>0.05)$.

During the 7 months before the COVID-19 outbreak, in the study group, there was no significant difference in the SE change of myopia, emmetropia, and hyperopia. The mean change of hypermetropic children was $-0.38 \pm 0.80$ $\mathrm{D}$, and the mean change of emmetropia and myopia was $-0.66 \pm 0.83 \mathrm{D}$ and $-0.63 \pm 0.90 \mathrm{D}(P=0.217)$. In January 2020, 122 out of 208 children were myopia. During the 7 months after COVID-19 outbreak, 178 children were myopia. No variation toward less myopia or more hyperopia was found.

Figure 1 shows the changes of ocular parameters before and during studying at home. The SE changes of right eyes were $0.60 \mathrm{D}(P<0.001)$. The SE changes between the control period and during studying at home were significantly different $(P<0.001)$. The changes of UCVA between two periods in right eyes were not significantly different $(P>0.05)$. Over the 7 months before outbreak, the mean value of axial elongation was $0.23 \pm 0.18 \mathrm{~mm}$ in the right eye, which was not significantly difference than $0.24 \pm 0.19 \mathrm{~mm}$ during outbreak $(P=0.37)$.

Before the COVID-19 outbreak, in the study group, there was no statistically significant difference in the

Table 1 The value of ocular characteristics of right eyes at baseline

\begin{tabular}{lllll}
\hline & Maximum & Minimum & Mean & $\begin{array}{l}\text { Standard } \\
\text { deviation }\end{array}$ \\
\hline UCVA & 1.00 & -0.10 & 0.17 & 0.22 \\
Axial length, mm & 25.60 & 20.05 & 23.08 & 0.91 \\
Spherical equivalent, D & 1.75 & -7.88 & -0.50 & 1.25 \\
\hline
\end{tabular}


Fig. 1 The changes of ocular parameters among control group, before and during COVID-19 pandemic study at home

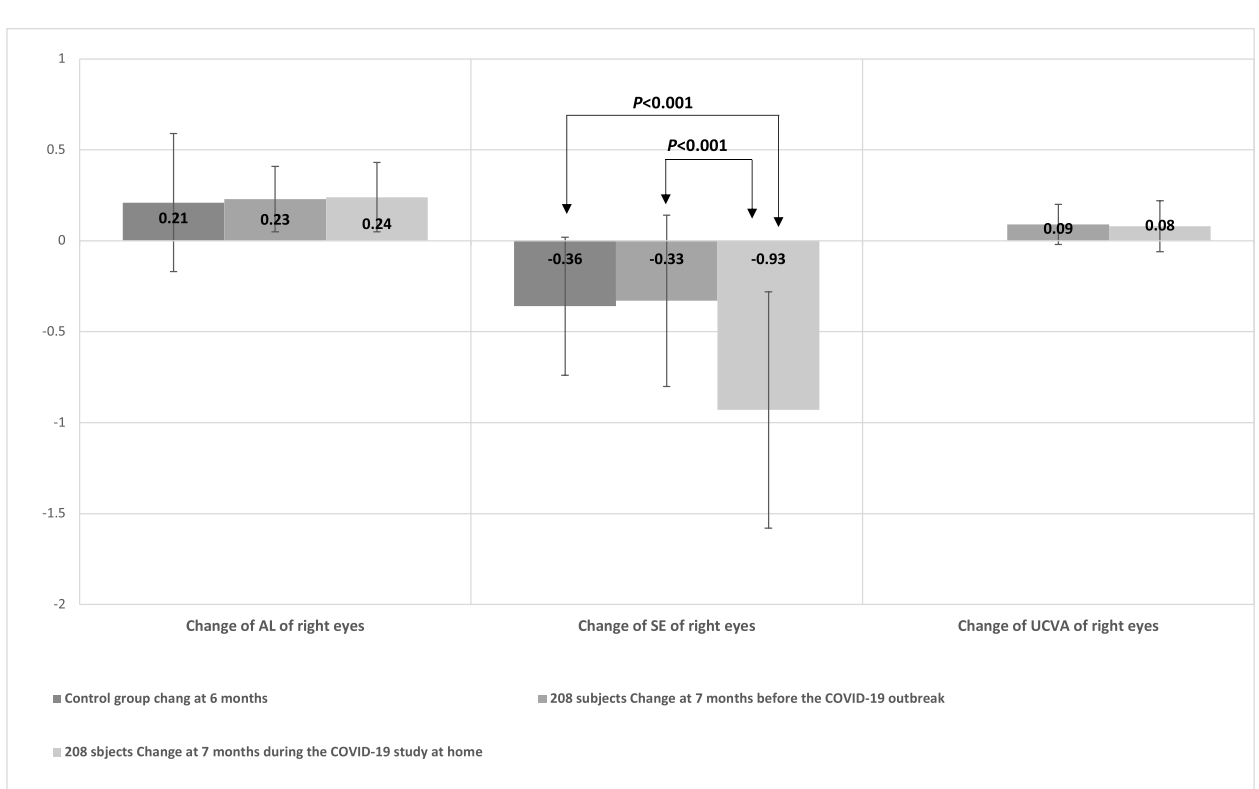

amount of time spent on various activities by myopic, emmetropic, and hyperopic children. The time spent on near work was $3.06 \pm 1.98 \mathrm{~h} /$ day in children with hyperopia, $3.45 \pm 2.82 \mathrm{~h}$ in children with emmetropia, and $3.32 \mathrm{~h}$ in children with myopia $(P=0.640)$. Children with myopia, emmetropia, and hyperopia spent $2.06 \pm 2.065 \mathrm{~h} /$ day, $1.90 \pm 1.36 \mathrm{~h} /$ day, and $1.83 \pm 2.03 \mathrm{~h} /$ day on outdoor activities, respectively $(P=0.348)$.

Compared with before COVID-19 outbreak, during studying at home, time spent in homework, and reading books increased from $1.46 \pm 0.98$ to $1.77 \pm 1.06 \mathrm{~h} /$ day. Time spent in other near work like painting and playing chess before and during COVID-19 pandemic were $0.38 \pm 0.47 \mathrm{~h} / \mathrm{d}$ ay and $0.45 \pm 0.6 \mathrm{~h} / \mathrm{day}$, respectively. Before COVID-19 outbreak, children spent $1.42 \pm 1.77 \mathrm{~h} /$ day in digital screen included using computer and mobile phone, with no online education. During COVID-19 outbreak, children spent $2.43 \pm 2.19 \mathrm{~h} /$ day in digital screen, and $1.94 \pm 1.05 \mathrm{~h} /$ day in online education. Compared with before COVID-19 outbreak, during studying at home, outdoor activities decreased from $1.75 \pm 1.52$ to $0.90 \pm 1.04 \mathrm{~h} / \mathrm{day}$. The details are presented in Fig. 2.

A binary logistic regression was employed to analyze the relations of the different factors associated with myopia, including the age, gender, baseline UCVA, baseline $\mathrm{AL}$, baseline SE, and the changes of time between before and after homework and reading, digital screen, other near work, as well as the change of time of online learning during the COVID-19, and the change of time of outdoor activities. The results showed that the difference of the SE's change was related to the baseline AL, the change of time of using digital screen, and the time of online learning (Fig. 3).

\section{Discussion}

It was reported that the rate of myopia among Chinese primary and middle school students increased by $11.7 \%$ in the first 6 months of the year [15]. However, data on myopia progression and mydriatic spherical equivalent in children during studying at home were lacking. As far as we know, this is the significant report analyzing the progression of myopia in children during studying at home. The mean SE change was - 0.9D in 7 months during studying at home. However, in the 7 months before the COVID-19, the mean myopia progression was $-0.3 \mathrm{D}$ and this difference was found to be statistically significant. Moreover, it was found that the difference of the mydriatic spherical equivalent changes was related to the children's baseline AL, longtime online leaning, and digital screen reading.

The control group about myopia progression in Chinese children reported that at the 6-month follow-up, the mean values of myopia progression was $-0.36 \mathrm{D}$, and mean value of axial elongation was $0.21 \mathrm{~mm}$ [21]. These results were similar as ocular parameter's change before the COVID-19 outbreak. Compared with the control group, the average 


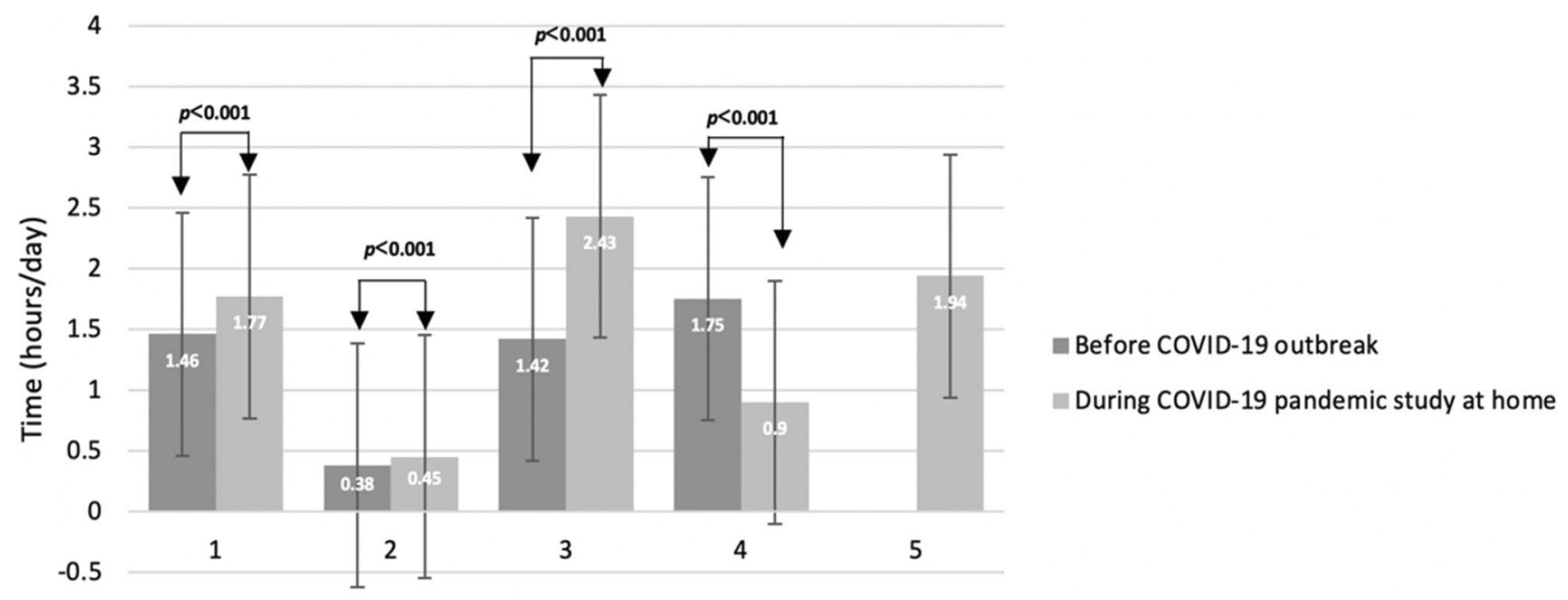

$-1$

1:Completing homework and reading books; 2:Other near work; 3:Using the digital screen; 4:Combined outdoor activities; 5:Online learning

Fig. 2 Time spent in near work and outdoor activities (hours/day) before and during studying at home

Fig. 3 Logistic regression SE's change analysis of the difference of the

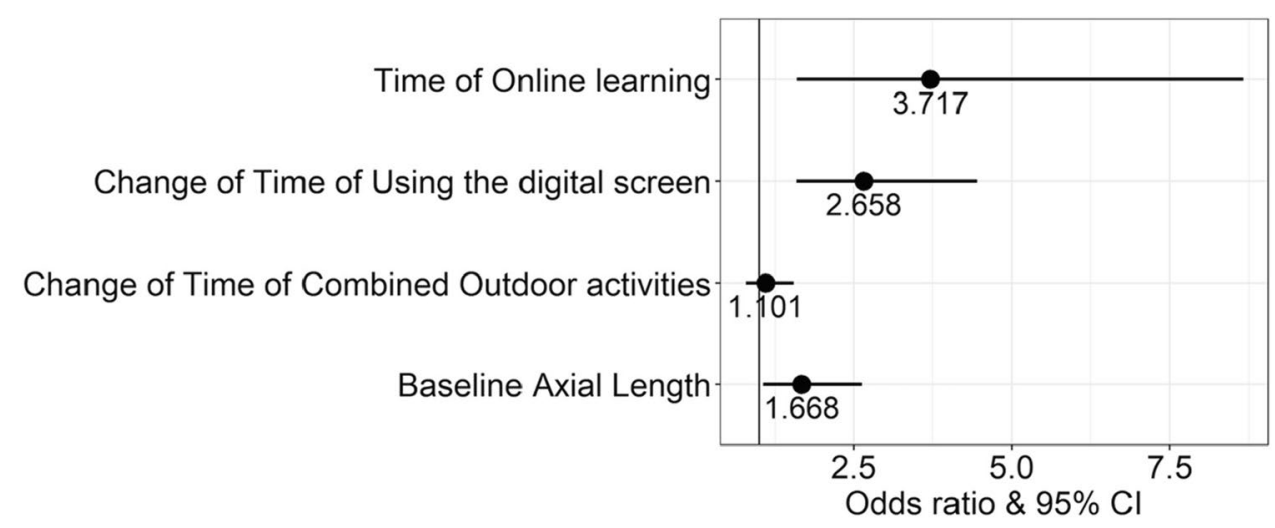

progression of SE during 7-month study at home was statistically significant (Fig. 2).

A summary of the myopia progression in previous studies is shown in Table 2. Our study found the myopia progression of $-0.3 \pm 0.5 \mathrm{D}$ in 7 months before the COVID19 what was similar to the previous studies of Chinese and foreign children showing myopia progression of $-0.3 \mathrm{D}$ in 6 months or $-0.6 \mathrm{D}$ in a year [22-28]. There were also some studies presenting higher myopia progression [25, 29-31]. We believe that this may be related to different genetic and environmental factors as well as differences in age and the size of refractive error in different studies. Compared with previous studies, we found that myopia progressed rapidly during studying at home [22-32]. The myopia progression was $-0.9 \mathrm{D}$ in 7 months during studying at home.
Our study showed that compared with before the COVID19, there was a significant change in SE, but no significant change in axial length and UCVA during the COVID-19 pandemic. We speculate that it may be transient myopia caused by accommodative spasm. More data and studies are needed to support this hypothesis in the future.

A large sample study of Chinese children found that myopia was significantly related to near work like continuous reading [33]. Near work was considered as a risk factors for myopia through increased accommodative demand [33]. Longtime near work causes the increase of prevalence and incidence of myopia in children [34, 35]. Long-term usage of electronic screens, near work, and limited outdoor myopia [36]. activities were associated with the onset and progression of 
Table 2 Key results and myopia progression from previous studies

\begin{tabular}{|c|c|c|c|c|c|c|}
\hline Reference & Location & Age, y & Sample size & $\begin{array}{l}\text { Duration, } \\
\text { months }\end{array}$ & Average myopia, D & Myopia progression, D \\
\hline Donovan L et al., 2012[22] & China & $6-12$ & 85 & 6 & NA & $\begin{array}{l}-0.31 \pm 0.25 \text { for summer } \\
-0.53 \pm 0.29 \text { for winter }\end{array}$ \\
\hline Fujiwara M et al., 2012[24] & Japan & $10-13$ & 92 & 6 & $-4.40 \pm 1.38$ & $\begin{array}{l}-0.35 \pm 0.04 \text { for summer } \\
-0.28 \pm 0.06 \text { for winter }\end{array}$ \\
\hline Cui D et al., 2013[32] & Denmark & $8-14$ & 235 & 6 & $-2.24 \pm 1.39$ & $-0.287 \pm 0.266$ \\
\hline Jane G et al., 2014[26] & USA & $6-12$ & 469 & 6 & $-2.54 \pm 0.84$ & $\begin{array}{l}-0.35 \pm 0.34 \text { for winter } \\
-0.14 \pm 0.32 \text { for summer }\end{array}$ \\
\hline Yu S et al., 2015[29] & China & $6-15$ & 900 & 6 & NA & $-0.56 \pm 0.37$ \\
\hline Clark et al., 2015[27] & USA & $6-15$ & NA & 12 & $-2.0 \pm 1.5$ & $-0.6 \pm 0.4$ \\
\hline Yam et al., 2018[30] & Hong Kong & $4-12$ & 109 & 12 & $-3.85 \pm 1.95$ & $-0.81 \pm 0.53$ \\
\hline Pei w, et al., 2018[31] & Taiwan & $6-7$ & 41 & 12 & NA & $-0.79 \pm 0.38$ \\
\hline Sacchi et al., 2019[25] & Italy & $5-16$ & 50 & 12 & $-2.63 \pm 2.68$ & $-1.09 \pm 0.64$ \\
\hline Larkin et al., 2019[28] & USA & $6-15$ & 98 & 12 & $-2.8 \pm 1.6$ & $-0.6 \pm 0.4$ \\
\hline Yun c et al., 2020[23] & China & $8-15$ & 144 & 12 & $-3.16 \pm 1.13$ & $-0.61 \pm 0.31$ \\
\hline
\end{tabular}

Digital screen time has been reported as a risk factor that could increase myopia [37]. During the COVID-19 pandemic, students were away from the school classroom and have the online education that greatly increased the digital screen time.

Due to kept at home, children and adolescents spent more time reading books, watching TV, playing video games, and using computers, tablets, and smartphones. During the COVID-19 pandemic, Canadians aged 15-49 spent $66 \%$ more time watching TV and $35 \%$ more playing video games than before the COVID-19 outbreak [38]. In South Korea, $79(81 \%)$ of 97 parents said their children's digital time had increased [39].

Previous studies have found that outdoor time has a protective effect on myopia onset [39-41]. During COVID-19 pandemic, the outdoor activities of children and teenagers have been reduced because of the various lockdown measures imposed on populations everywhere to contain to spread of the virus. Although the outdoors activities time decreased, our study did not show any relation between outdoor activity and myopia progression. We speculated that it could be due to the small sample size of this study that the results were different from those of previous studies.

Although it is the unique study investigating the COVID19 lifestyle impact on myopia development and progression in natural cohort of children, this study has several limitations. First is the selection bias due to a relatively small sample size. Secondly, the data on time and parameters of near work and outdoor activities were not directly measured, but were acquired by questionnaires, which may have led to recall bias. To minimize this bias, all questionnaires were completed by children and parents. Thirdly, previous studies [22, 26] found myopia progressed more slowly in summer than in winter. Handan has seasonal changes through the year. In our study, the two periods of observation were in different seasons, which may bias the outcomes. However, home isolation during the COVID-19 epidemic mainly occurred in the summer months, and our study found the myopia progression was greater in children during this period. This indicates that changes in living styles and learning environment due to the COVID-19 lead to greater myopia progression.

\section{Conclusion}

In summary, the nature cohort study in children revealed that the children were at risk of myopia progression during the COVID-19 pandemic study at home than 7 months before the outbreak. The myopia progression was related to the initial AL, the longtime of online learning, and the longtime of digital screen. Studying at home during COVID-19 may be posing a threat to the development and progression of myopia in children and adolescents, especially in East Asia, where the prevalence of myopia is high.

Author contribution All authors contributed to the study conception and design; data collection: Dandan Ma, Shifei Wei, Shi-Ming Li, Xiaohui Yang, Sujie Fan, Lihua Zhang; statistical expertise: Dandan $\mathrm{Ma}$; administrative, technical, and logistic support: Shi-Ming Li, Ningli Wang; analysis and interpretation: Kai Cao, Jianping $\mathrm{Hu}$; writing the article: Dandan Ma, Shifei Wei; critical revision of the article: Dandan Ma, Shifei Wei, Ningli Wang, Shi-Ming Li; final approval of the article: Dandan Ma, Shifei Wei, Shi-Ming Li, Xiaohui Yang, Kai Cao, Jianping Hu, Sujie Fan, Lihua Zhang, Ningli Wang. All authors reviewed the manuscript and agreed to be accountable for all aspects of the work.

Funding This work was supported by the Fund Sponsorship of the Capital Public Health Project (Z171100000417017), the Beijing Natural Science Foundation (JQ20029), the Capital health research and 
development of special (2020-2-1081), and the National Natural Science Foundation of China (82071000).

Data availability All data generated or analyzed during this study are included in this published article.

\section{Declarations}

Ethics approval and consent to participate This research followed the tenets of the Declaration of Helsinki and was approved by Beijing Tongren Hospital Ethical Committee. Informed consent was obtained from all subjects.

\section{Consent for publication Not applicable.}

Competing interests The authors declare no competing interests.

Open Access This article is licensed under a Creative Commons Attribution 4.0 International License, which permits use, sharing, adaptation, distribution and reproduction in any medium or format, as long as you give appropriate credit to the original author(s) and the source, provide a link to the Creative Commons licence, and indicate if changes were made. The images or other third party material in this article are included in the article's Creative Commons licence, unless indicated otherwise in a credit line to the material. If material is not included in the article's Creative Commons licence and your intended use is not permitted by statutory regulation or exceeds the permitted use, you will need to obtain permission directly from the copyright holder. To view a copy of this licence, visit http://creativecommons.org/licenses/by/4.0/.

\section{References}

1. Qiao C, Zhang H, He M et al (2020) Symptomatic COVID19 in eye professionals in Wuhan China. Ophthalmology 127(9):1268-1270

2. Johns Hopkins University and Medicine (2020) Coronavirus Resource Center https://coronavirus.jhu.edu/map.html. Accessed 10 Nov 2020

3. United Nations Educational, Scientific and Cultural Organization (2020) https://zh.unesco.org/themes/education-emergencies/coron avirus-school-closures. Accessed 1 Jan 2021

4. Wang G, Zhang Y, Zhao J et al (2020) Mitigate the effects of home confinement on children during the COVID-19 outbreak. The Lancet 395(10228):945-947

5. CCTV News (2020) Ministry of Education: national elementary and middle school network cloud platform opens for free use today. http://www.chinanews.com/sh/2020/02-17/9094648.shtml. Accessed 3 Mar 2020

6. Dolgin E (2015) The myopia boom. Nature 519(7543):276-278

7. Holden BA, Fricke TR, Wilson DA et al (2016) Global prevalence of myopia and high myopia and temporal trends from 2000 through 2050. Ophthalmology 123(5):1036-1042

8. Ding BY, Shih YF, Lin LL et al (2017) Myopia among schoolchildren in East Asia and Singapore. Surv Ophthalmol 62(5):677-697

9. Yotsukura E, Torii H, Inokuchi M et al (2019) Current prevalence of myopia and association of myopia with environmental factors among schoolchildren in Japan. JAMA Ophthalmol 137(11):1233-1239

10. He M, Zheng Y, Xiang F (2009) Prevalence of myopia in urban and rural children in mainland China. Optom Vis Sci 86(1):40-44
11. Congdon N, Wang Y, Song Y et al (2008) Visual disability, visual function, and myopia among rural chinese secondary school children: the Xichang Pediatric Refractive Error Study (X-PRES)report 1. Invest Ophthalmol Vis Sci 49(7):2888-2894

12. Li SM, Liu LR, Li SY et al (2013) Design, methodology and baseline data of a school-based cohort study in Central China: the Anyang Childhood Eye Study. Ophthalmic Epidemiol 20(6):348-359

13. Wei S, Sun Y, Li SM et al (2019) Visual impairment and spectacle use in university students in Central China: the Anyang University Students Eye Study. Am J Ophthalmol 206:169-175

14. Pellegrini M, Bernabei F, Scorcia V et al (2020) May home confinement during the COVID-19 outbreak worsen the global burden of myopia? Graefes Arch Clin Exp Ophthalmol 258(9):2069-2070

15. CHINADAILY (2020) Myopia rate of students rises 11.7 percent in H1: ministry http://www.chinadaily.com.cn/a/202008/27/ WS5f477763a310675eafc55ec5.html. Accessed 13 Nov 2020

16. Liang YB, Friedman DS, Wong TY et al (2009) Rationale, design, methodology, and baseline data of a population-based study in rural China: the Handan Eye Study. Ophthal Epidemiol 16(2):115-127

17. Li SM, Liu LR, Li SY et al (2013) Design, methodology and baseline data of a school-based cohort study in Central China: the Anyang Childhood Eye Study. Ophthal Epidemiol 20(6):348-359

18. Shi-Ming L, He L, Si-Yuan L et al (2015) Time outdoors and myopia progression over 2 years in Chinese children: the Anyang Childhood Eye Study. Invest Ophthalmol Vis Sci 56(8):4734-4740

19. Atchison DA, Li SM, Li H et al (2015) Relative peripheral hyperopia does not predict development and progression of myopia in children. Invest Ophthalmol Vis Sci 56(10):6162-6170

20. Panel AOAC (2008) Care of the patient with hyperopia. http:// citeseerx.ist.psu.edu/viewdoc/download?doi=10.1.1.180.2661\& rep $=$ rep $1 \&$ type $=$ pdf. Accessed 30 Oct 2015

21. Shifei W, Shi-Ming Li, Wenzai An et al (2020) Safety and efficacy of low-dose atropine eyedrops for the treatment of myopia progression in Chinese children: a randomized clinical trial. JAMA Ophthalmol 138:1178-1184

22. Donovan L, Sankaridurg P, Ho A et al (2012) Myopia progression in Chinese children is slower in summer than in winter. Optom Vis Sci 89(8):1196-1202

23. Chen Y, Drobe B, Zhang C et al (2020) Accommodation is unrelated to myopia progression in Chinese myopic children. Sci Rep 10(1):12056

24. Fujiwara M, Hasebe S, Nakanishi R et al (2012) Seasonal variation in myopia progression and axial elongation: an evaluation of Japanese children participating in a myopia control trial. Jpn J Ophthalmol 56(4):401-406

25. Sacchi M, Serafino M, Villani E et al (2019) Efficacy of atropine $0.01 \%$ for the treatment of childhood myopia in European patients. Acta Ophthalmol 97(8):e1136-e1140

26. Gwiazda J, Deng L, Manny R et al (2014) Seasonal variations in the progression of myopia in children enrolled in the correction of myopia evaluation trial. Invest Ophthalmol Vis Sci 55(2):752-758

27. Clark TY, Clark RA (2015) Atropine $0.01 \%$ eyedrops significantly reduce the progression of childhood myopia. J Ocul Pharmacol Ther 31(9):541-545

28. Luke Larkin G, Tahir A, Epley KD et al (2019) Atropine 001\% eye drops for myopia control in American children: a multiethnic sample across three US sites. Ophthalmol Ther 8(4):589-598

29. Yu S, Diao H, Zeng J (2015) Analysis of the prevalence and situation of myopia in adolescents from South China. Eye Sci 30(2):53-55

30. Yam JC, Li FF, Zhang X et al (2020) Two-year clinical trial of the Low-Concentration Atropine for Myopia Progression (LAMP) Study: phase 2 report. Ophthalmology 127:910-919 
31. Wu PC, Chen CT, Lin KK et al (2018) Myopia prevention and outdoor light intensity in a school-based cluster randomized trial. Ophthalmology 125(8):1239-1250

32. Cui D, Trier K, Munk Ribel-Madsen S (2013) Effect of day length on eye growth, myopia progression, and change of corneal power in myopic children. Ophthalmology 120(5):1074-1079

33. Saw SM, Hong CY, Chia KS et al (2001) Nearwork and myopia in young children. Lancet 357(9253):390

34. Li SM, Li SY, Kang MT et al (2015) Near work related parameters and myopia in Chinese children: the Anyang Childhood Eye Study. PLoS One 10(8):e0134514

35. Saw SM, Chua WH, Hong CY et al (2002) Nearwork in earlyonset myopia. Invest Ophthalmol Vis Sci 43(2):332-339

36. French AN, Morgan IG, Mitchell P et al (2013) Risk factors for incident myopia in Australian schoolchildren: the Sydney adolescent vascular and eye study. Ophthalmology 120:2100-2108

37. Lanca C, Saw S-M (2020) The association between digital screen time and myopia: a systematic review. Ophthalmic Physiol Opt 40(2):216-229

38. Wong CW, Tsai A, Jonas JB et al (2021) Digital screen time during COVID-19 pandemic: risk for a further myopia boom? Am J Ophthalmol 223:333-337
39. Deng L, Pang Y (2019) Effect of outdoor activities in myopia control: meta-analysis of clinical studies. Optom Vis Sci 96(4):276-282

40. Cao K, Wan Y, Yusufu M et al (2019) Significance of outdoor time for myopia prevention: a systematic review and metaanalysis based on randomized controlled trials. Ophthalmic Res 63(2):97-105

41. Xiong S, Sankaridurg P, Naduvilath T et al (2017) Time spent in outdoor activities in relation to myopia prevention and control: a meta-analysis and systematic review. Acta Ophthalmol 95(6):551-566

Publisher's note Springer Nature remains neutral with regard to jurisdictional claims in published maps and institutional affiliations. 